\title{
Factores de riesgo para dificultades en el aprendizaje de la lecto-escritura
}

\author{
Rosas Laureano C, Beteta García N, Granados Ramos D
}

\section{RESUMEN}

Se revisaron 55 expedientes de niños con dificultades en el proceso de lecto-escritura. Se establecieron factores de riesgo perinatal, presencia y severidad de dificultades en el aprendizaje de la lecto-escritura. El 85\% de niños con factores de riesgo perinatal presentaron dificultades en el proceso de la lecto-escritura. La detección temprana de factores de riesgo perinatal y establecimiento de estrategias de intervención oportuna contribuirán a la disminución de secuelas en etapas escolares.

Palabras clave: factores de riesgo perinatal, lecto-escritura, aprendizaje.

\section{Risk factors for learning difficulties in the reading/writing}

\begin{abstract}
Fifty five files of children with difficulties in the reading/writing process were reviewed. The perinatal risk factors, the presence and severity of difficulties in the learning of the reading/writing were established. $85 \%$ of children with perinatal risk factors presented difficulties in the reading/writing process. The early detection of perinatal risk factors and the establishment of opportune strategies of intervention will contribute to the diminution of sequelaes in school stages.
\end{abstract}

Key words: perinatal risk factors, reading-writting, learning.

Correspondencia: Rosas Laureano C.Vicente Guerrero \# 2 Patria Libre 96718. Minatitlán Ver, México. E-mail: cbyasiempre_04@hotmail.com

$\mathrm{E}$ 1 desarrollo del lenguaje y el pensamiento en la ducación básica, constituyen la base sobre la cual pueden alcanzarse elevadas competencias psicolingüísticas. El aprendizaje de la lecto-escritura es una tarea lingüística y cognitiva compleja que puede resultar negativamente afectada por un gran número de factores biológicos y ambientales en etapas tempranas del desarrollo 1 .

Los factores de riesgo; son atributos, características o circunstancias que incrementan la probabilidad de desarrollar un daño a la salud. Pueden ser indicadores predictivos observables o identificables de algún hecho o proceso mórbido².

Los factores de riesgo para un proceso mórbido pueden ser de tipo biológico, ambiental, comportamiento o estilo de vida, relacionados con la atención a la salud, socioculturales o económicos ${ }^{2}$. En el caso de dificultad en el aprendizaje se deben considerar los factores de riesgo prenatales, perinatales y posnatales que contribuyen al establecimiento de dificultades para la adquisición y desarrollo de habilidades cognoscitivas como la lecto-escritura.
Algunas investigaciones han demostrado que el diagnóstico temprano de los precursores de la lectura en niños preescolares, antes de comenzar su aprendizaje formal, puede ser muy útil para establecer estrategias de intervención en poblaciones de riesgo de padecer dificultades en la adquisición de la lectoescritura $^{3}$.

En los dos primeros años de educación primaria se inicia el aprendizaje formal del proceso de lecto-escritura. En ese momento los niños se enfrentan a las dificultades en la adquisición de la lecto-escritura.

Los casos que no son detectados por sus padres o profesores son promovidos a los grados superiores presentando deficiencias importantes en su proceso educativo llegando a reprobar y desertar en los niveles educativos medios o superiores.

Se ha demostrado que los niños con factores de riesgo perinatal desarrollan dificultades de procesamiento sensorial, problemas de aprendizaje, dislexia, hiperactividad y trastornos de 
atención en las etapas preescolar y escolar ${ }^{4-7}$. Algunos investigadores opinan que los factores de riesgo perinatal aparecen y operan aditivamente; es decir, a mayor número de factores de riesgo más severas serán las secuelas que deriven de los mismos ${ }^{8}$.

\section{OBJETIVO}

Analizar y describir los factores de riesgo perinatales en expedientes de escolares con dificultades en la adquisición de la lecto-escritura.

\section{MATERIAL Y MÉTODOS}

Se realizó una investigación retrospectiva, descriptiva con 55 expedientes clínicos de niños de 6 a 11 años de edad del primero al quinto año de educación primaria, que presentaron dificultades en la adquisición de la lecto-escritura, que asistieron a evaluación al laboratorio de psicobiología en Xalapa, Veracruz, 19 casos fueron del género femenino y 36 masculinos.

De los expedientes clínicos se recabaron los datos de factores de riesgo perinatal de la madre y del niño. Se calificó el grado de severidad de factores de riesgo perinatales de acuerdo a los criterios del grupo de estudios del nacimiento ${ }^{9,10}$.

Se estableció el nivel de lecto-escritura en el que se encontraban los niños como adquisición, proceso de adquisición y proceso de adquisición tardía. Los niños que se encontraban en adquisición, fueron aquellos niños que ya habían aprendido a leer y escribir; no obstante, presentaban dificultades en el proceso como: omisiones, sustituciones e inversiones de fonemas; los que se clasificaron en proceso de adquisición fueron aquellos niños que estaban aprendiendo a leer y escribir en etapas esperadas para su edad; no obstante, presentaban problemas en la adquisición porque cursaban con rezago comparados con sus compañeros de grupo; por último, los niños en proceso de adquisición tardía fueron los que a edades mucho mayores a la esperada, aún estaban aprendiendo a leer y a escribir, continuaban en el primer o segundo grado de educación primaria.

Se estableció el nivel de severidad (normal, leve, moderada y severa) de las dificultades en el proceso de adquisición de la lecto-escritura a través de los resultados de evaluaciones psicopedagógicas, de acuerdo a lo esperado para la edad y grado escolar que estaba cursando cada uno de los niños. Se analizaron los datos con análisis de varianza de una vía con el programa JMP versión 5.1.

\section{RESULTADOS}

En el análisis de factores de riesgo perinatal 13 casos (.24) correspondieron al nivel bajo, 11 (.20) al nivel medio y 31 (.56) al nivel alto.
En el nivel de lecto-escritura en que se encontraban al momento de la evaluación, ocho casos (.14) ya habían aprendido a leer; sin embargo, presentaban dificultades, 40 casos (.73) estaban en el proceso de adquisición, pero mostraban dificultades en el mismo y siete casos (.13) estaban en un proceso de adquisición tardío.

En la revisión de severidad en el proceso de lecto-escritura de acuerdo a lo esperado para la edad y nivel de adquisición, ocho casos (.14) estaban dentro de lo normal, 12 casos (.22) con retraso leve, 22 casos (.40) con retraso moderado y 13 casos (.23) con retraso severo gráfica 1.

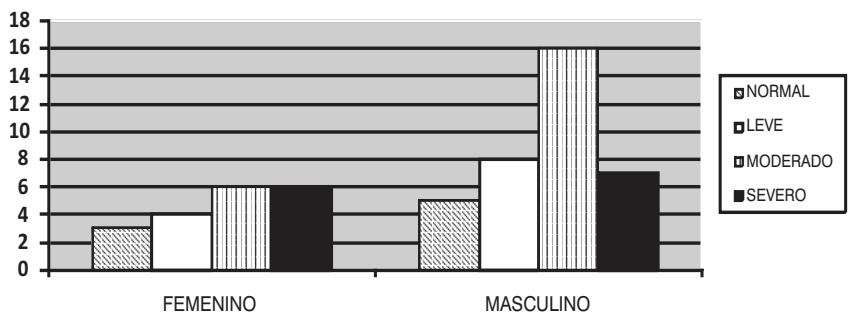

Gráfica 1. Nivel de severidad en el proceso de lecto-escritura.

En el análisis estadístico se encontraron diferencias significativas al comparar los factores de riesgo perinatal y presencia o no de dificultades en el proceso de adquisición de la lecto-escritura $(\mathrm{f}=4,2168, \mathrm{p}<0.04)$ gráfica 2.

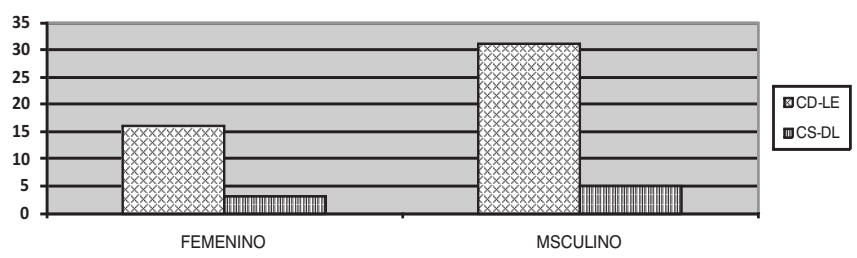

CD-LE (casos con dificultades en lecto-escritura), SD-LE (casos sin dificultades en lecto-escritura).

Gráfica 2. Proceso de lecto-escritura por género.

Al comparar la presencia o no de factores de riesgo perinatales y el género, así como los factores de riesgo perinatal por severidad con las dificultades en el proceso de lecto-escritura no se encontraron diferencias significativas.

En los casos con factores de riesgo perinatal alto (FRA) se encontraron 10 con dificultades moderadas en la lecto-escritura, respecto a las dificultades severas y para el nivel normal o esperado se contabilizaron ocho casos en ambas situaciones, por último se refiere a seis casos con dificultades leves.

En los casos con factores de riesgo perinatal medio (FRM) seis presentaron dificultades moderadas, tres severas, un caso leve y uno normal.

Por último, en los casos con factores de riesgo perinatal bajo (FRB) seis presentaron dificultades moderadas, cinco leves, 
dos severas y no hubo casos con adquisición normal o esperada para la edad y el grado escolar que estaban cursando tabla 1.

Tabla 1. Factores de riesgo perinatal y severidad en lecto-escritura

\begin{tabular}{c|c|c|c}
\hline \multirow{2}{*}{$\begin{array}{c}\text { Severidad en } \\
\text { lecto-escritura }\end{array}$} & \multicolumn{3}{|c}{ Factores de riesgo perinatal } \\
\cline { 2 - 4 } & Bajo & Medio & Alto \\
\hline Normal & 0 & $1(.02)$ & $7(.13)$ \\
Leve & $5(.09)$ & $1(.02)$ & $6(.11)$ \\
Moderado & $6(.11)$ & $6(.11)$ & $10(.18)$ \\
Severo & $2(.04)$ & $3(.05)$ & $8(.14)$ \\
Total & $13(.24)$ & $11(.20)$ & $31(.56)$ \\
\hline
\end{tabular}

Distribución de casos por factores de riesgo perinatal y dificultades de lecto-escritura en escolares.

\section{DISCUSIÓN}

Es necesario contar con más casos para que se analicen grupos homogéneos para el género, grado y grupos de edad. No obstante, este análisis nos permitió observar que el $85 \%$ de los niños que presentaron factores de riesgo perinatal, mostraron dificultades en el proceso de adquisición de la lectoescritura, lo cual no se pudo constatar al realizar comparaciones por grupos de severidad.

El mayor número de casos correspondió al género masculino lo cual concuerda con las investigaciones donde se han reportado mayor número de dificultades de aprendizaje en el género masculino ${ }^{10}$. Es necesario revisar más expedientes de diferentes servicios donde acuden a evaluación o intervención casos con dificultades de aprendizaje, para constatar si la frecuencia es mayor en el género masculino.

Nuestros datos concuerdan con las investigaciones de Duarte, Glasson, y Crepeau en cuanto a la presencia de factores de riesgo en la etapa perinatal predice la estructuración de secuelas sensoriales, motoras, cognoscitivas y conductuales que son detectadas en la educación básica.

\section{CONCLUSIÓN}

Es importante resaltar la trasedencia de establecer estrategias tempranas de prevención de factores de riesgo en etapa perinatal y brindar intervención temprana a los casos que presenten dichos factores de riesgo, con el objeto de disminuir la estructuración de secuelas y severidad de las mismas pues las dificultades de aprendizaje son detectadas hasta que el niño ingresa al medio escolar e inicia el aprendizaje de habilidades importantes para su desarrollo escolar futuro como son lectoescritura y nociones lógico-matemáticas.

\section{REFERENCIAS}

1. Beltrán Llera J, López Escribano C, Rodríguez E. Precursores tempranos de la lectura. Actas del Primer Congreso Nacional de Lingüística Clínica, 2006; 2.

2. Castillo Salgado C. Manual sobre el enfoque de riesgo en la atención materno infantil. Washington, DC: OPS, 1999: 342.

3. López Escribano, C, Beltrán J. Early predictor of reading in three groups of native Spanish speakers: Spaniards, Gypsies, and Latin Americans. Span J Psychol 2009; 12,1:84-95.

4. Duarte Martins M, Factores de riesgo para la calidad del ambiente en una población de niños nacidos en la ciudad de Pelotas, RS, Brasil, Tesis de Belgrano, Facultad de Humanidades, Doctorado en Psicología Clínica, 2002; 9, 1-60.

5. Glasson EJ. perinatal factors and the development of autism: a population study. Archiv Gen Psychi 2004; 61: 618-27.

6. Dean RS, Davis AS. Relative risk of prenatal complications in common childhood disorders. School Psychology Quarterly 2007; 22:1;13-25.

7. Crepeau Hobson F. The relationship between perinatal risk factors ans sensory processing difficulties in preschool children, J Dev Phys Disabil 2009;21:315-28.

8. Taylor E, Rogers JW. Practitioner review: early adversity and developmental disorders. J Child Psychol Psychi 2005;46:5,451-67.

9. PreviGen (2005), http://www.gen.org.mx/home.html

10. Rutter M. Gender differences in reading difficulties: findings from four epidemiology studies. J Am Med Ass 2004; 291: 2007-12. 